NASA Technical Memorandum 83634

AIAA-84-0991

\title{
Vibration and Flutter of Mistuned Bladed-Disk Assemblies
}

Krishna Rao V. Kaza and Robert E. Kielb

Lewis Research Center

Cleveland, Ohio

Prepared for the

Twenty-fifth Structures, Structural Dynamics and Material Conference cosponsored by the AIAA, ASME, ASCE, and AHS

Palm Springs, California, May 14-16, 1984 


\title{
VIBRATION AND FLUTTER OF MISTUNED BLADED-DISK ASSEMBLLIES
}

\author{
Krishna Rao V. Kaza* and Robert E. Kielb** \\ National Aeronautics and Space Administration \\ Lewis Research Center \\ Cleveland, Ohio 44135
}

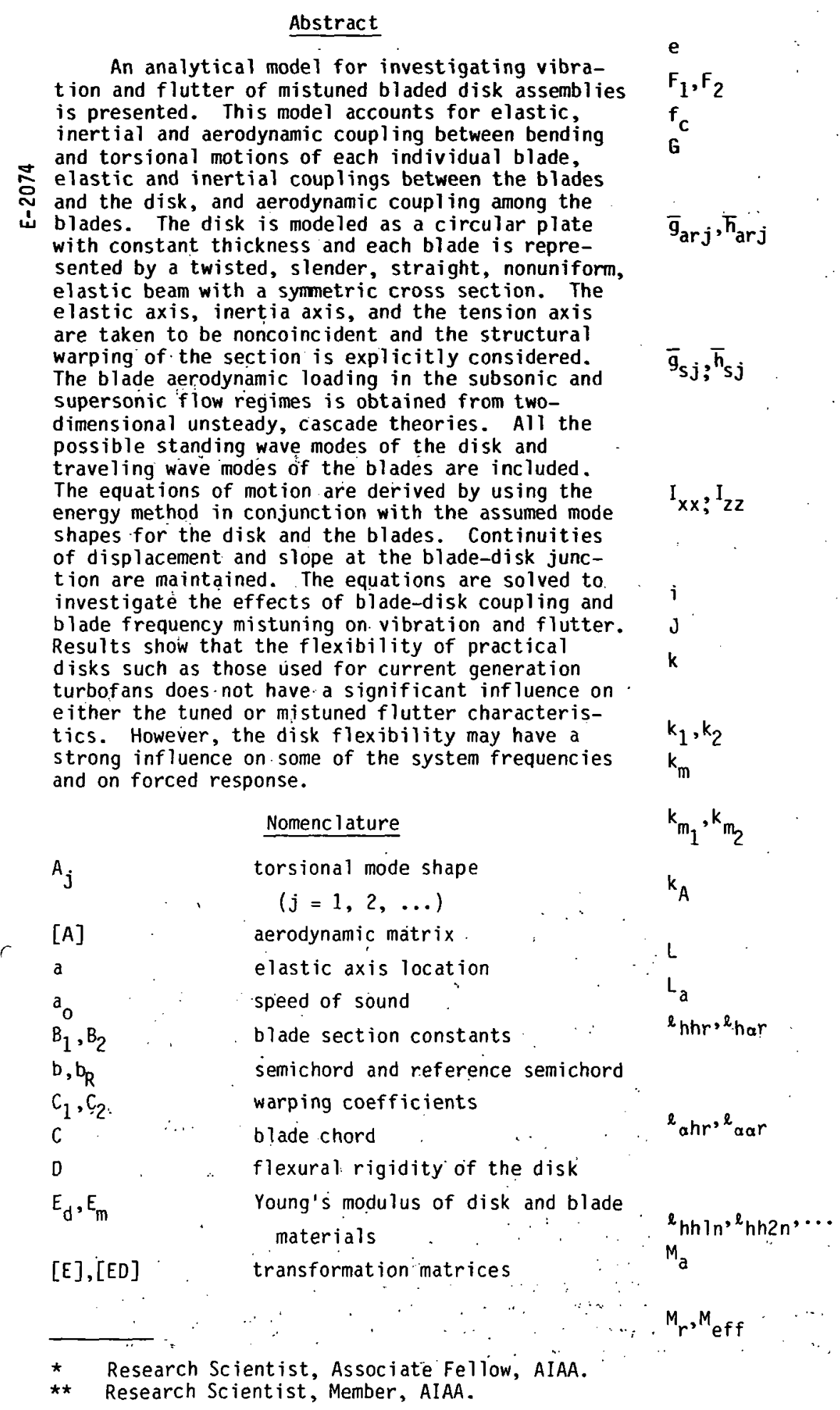

\section{Abstract} with constant thickness and each blade is repre(wisted, slender, straight, nonuniform, elastic axis, inertia axis, and the tension axis are taken to be noncoincident and the structural warping of the section is explicitly considered. blade aerodynamic loading in the subsonic and imersonic flow regimes is obtained from twopossible standing wave modes of the disk and traveling wave modes of the blades are included. The equations of motion are derived by using the investigate the effects of blade-disk coupling and ade frequency mistuning on vibration and flutter. Rults show that the flexibility of practical sh as those used for current generation inever, the disk flexibil strong influence on some of the system frequencies and on forced response.

$$
(j=1,2, \ldots)
$$

elastic axis location

of sound

semichord and reference semichord warping coefficients

blade chord

Young's modulus of disk and blade

$$
\text { materials }
$$

transformation matrices $f_{c}$

$\bar{g}_{a r j}, \bar{h}_{a r j}$

$\bar{g}_{s j}, \bar{h}_{s j}$

$I_{x x}, I_{z z}$

$k_{1}, k_{2}$

$k_{m_{1}}, k_{m_{2}}$

$k_{A}$

a

${ }^{\ell}$ hhr, $\ell_{\text {har }}$

$e_{\alpha h r}, l_{\alpha \alpha r}$

$e_{h h 1 n}, e_{h h 2 n}, \cdots$ M mass and elastic axis offset quantities defined in Eq. (13) function defined in Eqs. (9) shear modulus of elasticity of blade material

complex amplitudes of generalized coordinates out of and in the plane of rotation of the $r^{\text {th }}$ interblade phase angle mode complex amplitudes of generalized coordinates out of and in the plane of rotation of the $s^{\text {th }}$ blade

blade bending moment of inertia about the centroidal major and minor axes

$$
\sqrt{-1}
$$

torsional constant

reduced frequency, $\omega_{0} b / V_{\text {eff }}=$ $\omega_{0} b / M_{\text {eff }}{ }^{a} 0$

quantities defined in Eq. (13)

polar mass radius of gyration about elastic axis

mass radii of gyration about $x$ and $z$ axes

polar radius of gyration about elastic axis

blade length, $\left(R_{T}-R_{H}\right)$

lift per unit blade span

lift coefficients due to plunging and pitching motions in the $r^{\text {th }}$ cascade mode

moment coefficients due to plunging and pitching motions in the $r^{\text {th }}$ cascade mode coefficients defined in Eq. (17) aerodynamic moment per unit blade span

relative and effective Mach numbers 


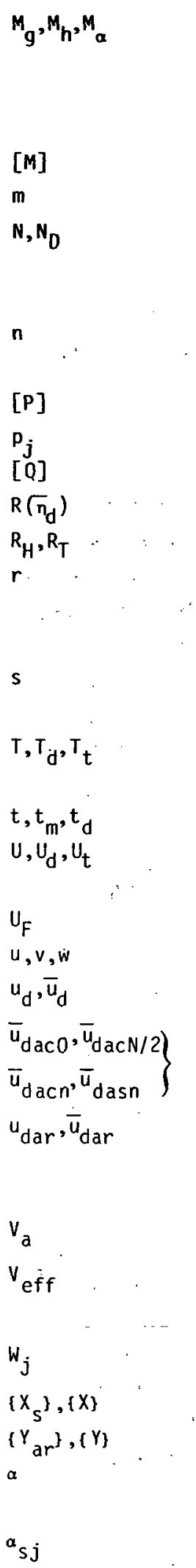

number of modes retained. in bending motions out of and in the plane of rotation and in torsion

inertia matrix

mass per unit blade length

number of blades in cascade and. number of. nodal diameters of the disk

integer specifying disk nodal diameter $\left(=1,2, \ldots, N_{D}\right)$

stiffness matrix of the system quantity defined in Eq. (11)

matrix defined in Eq. (29)

mode shape function of the disk

disk and blade radii

radial coordinate and also integer specifying cascade mode $(=0,1,2, \ldots, N-1)$

integer specifying blade

$(=0,1,2, \ldots, N-1)$

: kinetic energy of blade, disk and system

time, thickness of blade and disk strain energy of blade, disk and system

blade foreshortening

deformations of elastic axis

disk deflection, $\bar{u}_{d}=u_{d} / R_{H}$

amplitude of disk deflection in zero, $N / 2$, and $n^{\text {th }}$ nodal diameter cosine and sine modes

amplitude of disk deflection in the $r^{\text {th }}$ traveling mode of the disk, $\bar{u}_{\text {dar }}=u_{\text {dar }} / R_{H}$

axial velocity

effective velocity, $\sqrt{v_{a}^{2}+\Omega^{2} r^{2}}$ $\cos \left[90-\xi-\tan ^{-1} \cdot v_{a} / \Omega r\right]$ bending mode shape, $j=1,2, \ldots$.

column matrices

column matrices

twisting deformation positive about the $(-y)$ axis

complex amplitude of generalized coordinate in torsion of the $s^{\text {th }}$ blade $(j=1,2, \ldots)$

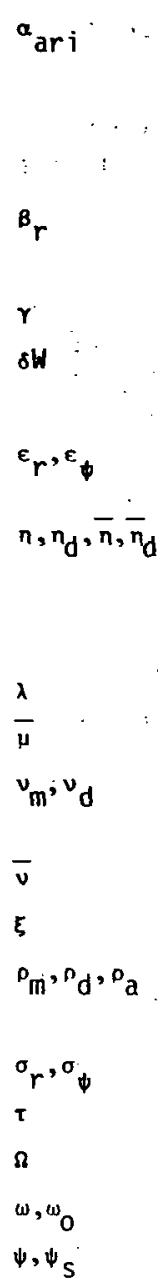

complex amplitude of generalized coordinate in torsion of the $r^{\text {th }}$ interblade phase angle mode of tuned cascade interblade phase angle in the $r^{\text {th }}$ mode nondimensional eigenvalue, $\left(\omega / \omega_{0}\right)^{2}$ virtual work by aerodynamic loading

strain components, Eq. (20)

blade and disk coordinates,

$$
\begin{aligned}
& n=r-R_{H}, n_{d}=r, \bar{n}=n / L ; \\
& \bar{n}_{d}=n_{d} / R_{H}
\end{aligned}
$$

warping function

reàl part of eigenvalue

Poisson's ratio of blade and disk materials

imaginary part of eigenvalue pretwist angle

densities of blade and disk: materials and air stress components, Eq. (20). nondimensional time, $\omega_{0} t$ rotational speed

frequency and reference frequency angular coordinate of disk and of the $s^{\text {th }}$ blade

\section{Introduction}

Vibration, aeroelastic stability and response of bladed-disk assemblies employed in modern aircraft turbofan engines have been among the most difficult analysis problems encountered. The blades are thin, and can have a considerable amount of built-in pretwist. As a consequence of pretwist and rotation, the blade natural modes involve coupling between the in-plane (the plane of rotation) and out-of-plane bending and the torsional motions. Another consequence of rotation is that the blades experience steady-state equilibrium loads which in turn require either an explicit or implicit consideration of geometric nonlinear theory of elasticity to adequately model the blade behavior.

Additionally, the individual blades are structurally coupled through the disk and of ten through a variety of other connecting parts such as shrouds, dampers, and lacing wires. These problems are further compounded by the presence of small differences between the individual blades, known as structural mistuning. 
Unlike the case of a fixed-wing, the flow Mach number varies along the blade span and involves unsteady subsonic, transonic, and supersonic flow regimes. The flow field is three-dimensional and also involves aerodynamic interference between adjacent blades within a stage as well as from upstream and downstream stators and rotors. Furthermore, there may be small differences in the flow between the individual blades, known as aerodynamic mistuning.

Because of the complexities mentioned above, the research work in the subject area has been proceeding with simple but progressively refined mathematical models. Vibration of the bladed-disk assemblies with structural coupling have been studied in many publications, for example Refs. 1 to 4. Flutter and response using single degree-offreedom blade models without structural coupling between blades, including mistuning, are addressed in Refs. 5 to 8 . Refs. 9 to 14 have considered flutter of tuned assemblies with rigid disks. A finite element formulation was presented in Ref. 15 to study flutter of bladed-disk systems. Refs. 16 and 17 investigated the effects of mistuning on cascade flutter and response in incompressible, subsonic and supersonic flow regimes with a rigid disk and a typical section model in which each blade has two degrees-of-freedom, one bending and one torsion. Effects of blade mistuning on flutter were also investigated in Refs. 18 to 19 . Furthermore, the single degree-offreedom typical section model used in Ref. 5 was modified in Ref. 20 to include disk flexibility and a sub- sonic aerodynamic theory was used to investigate flutter. The two degree-of-freedom typical sec-tion model used in Refs. 16 and 17 was refined in Ref. 21 to include disk flexibility and to study flutter and response.

. The typical section model used in Refs. 16 , 17 and 21 was found to be adequate to elicit physical understanding of mistuning effects. Parametric studies indicated that structural mistuning has the potential to significantly raise the flutter speed of an advanced turbofan. This potential may be utilized to eliminate the shrouds which have an adverse effect on aerodynamic performance. To obtain more accurate flutter boundaries, including the effects of mistuning, the typical section model was replaced by a beam model with a rigid disk in Ref. 22. The beam model was further extended in Ref. 23 to include blade sweep in order to investigate the possibility of classical flutter in advanced turboprops.

A logical extension to the present state of the literature is to refine the structural model used in Ref. 22 to include disk flexibility by treating the disk as a circular plate. The purpose of the research in the present paper is to combine such a structural model for the disk with a beam model for each blade in order to study the effects of coupling between disk and blade modes and of blade frequency mistuning on vibration and flutter.

The beam model used in Ref. 22 is similar to the one used in Ref. 24, but there the effect of warping was only partially considered in an implicit manner. In Ref. 25, the present authors found that the partial inclusion of warping in calculating uncoupled torsional frequencies and mode shapes of twisted rotating turbofan blades is not adequate and, hence, they modified the model to include all the warping effects. This modified beam model will be used herein. 'The unsteady, two-dimensional, cascade, aerodynamic loads are calculated by Smith's theory (26) in sybsonic flow and Adamczyk and Goldstein's theory $(27)$ in supersonic flow with subsonic leading edge:

The equations of motion are derived by using an energy method in conjunction with assimed mode shapes for the disk and for each blade. Continuity of displacements and slopes at the blade-disk junction is maintained. The resulting equations are cast in the form of a standard complex eigenvalue. problem from which the vibration and aeroelastic stability characteristics are determined. A computer program is developed to form and to solve the complex eigenvalue program. In order to have checks at least for some special cases, the equations and the computer program developed in Ref. 22 form special cases of the present ones.

This paper presents an analytical model, the development of the equations of motion and computer program, and some selected results for an advanced fan stage.

\section{Theory}

The motion of a bladed-disk assembly with or without mistuning $c$ an be expressed in terms of either a standing or traveling wave form. Since the stability is independent of the wave representation, consideration of either of the representations is adequate to describe the motion. In most of the published literature $(5,16,21,22)$ dealing with blade flutter of turbomachines, the traveling wave modes (also called interblade phase angle modes) are preferred because the unsteady aerodynamic loads are expressed in these modes. This is in contrast to the conventional flutter analysis of nonrotating wings and vibration of disks in which the standing wave modes are used. However, the present formulation uses both types of modes because the development includes both the blade and the disk flexibility and also is based on the development in Ref. 22. Hence, it will be necessary to understand both the traveling and standing wave mode representations and the relation between them.

To understand the traveling wave modes, consider the vibratory bending motion of each blade of an N-bladed rotor with a rigid disk: For illustration purposes, consider only one bending mode for each blade. Then the cascade has $N$ degrees of freedom. The motion of the system $c$ an be expressed in terms of traveling wave modes each of which is characterized by the phase angle $\beta_{r}$ between the adjacent blades. The angle is commonly called the interblade phase angle. The phase angle is restricted to $\mathrm{N}$ discrete values, $\mathrm{B}_{r}=2 \pi r / \mathrm{N}$ where the wave number index $r=0,1,2, \ldots(N-1)$. In each of these modes, all blades move with the same amplitude and every adjacent pair moves with the same interblade phase angle.

To understand the standing wave modes and the relation between the traveling and standing wave modes, consider the disk bending deflection perpendicular to the plane of the disk in the traveling wave form as 


$$
\bar{u}_{d}=\frac{u_{d}}{R_{H}}=\sum_{r=0}^{N-1} R\left(\bar{n}_{d}\right) \bar{u}_{d a r} e^{i\left(r_{\phi}+\frac{\omega}{\omega_{0}} \tau\right)}
$$

Theoretically, the wave numbers for an elastic disk can have values up to infinity. However, if. $N$ blades are attached to the disk and if the number of traveling wave modes are restricted to $\mathrm{N}$, then the wave number index is also limited to $(\mathrm{N}-1)$. Thus, the disk with the $\mathrm{N}$ attached blades can be characterized by $\mathrm{N}$ traveling wave modes. Assuming simple harmonic motion, the real part of Eq. (1), can be rearranged to give the disk deflection in the standing wave form as

$\bar{u}_{d}=R\left(\bar{n}_{d}\right)\left[\bar{u}_{d a c 0}+\bar{u}_{d a c N / 2} \cos \frac{N}{2} \psi\right.$

$$
\begin{aligned}
& +\sum_{n=1}^{N_{D}}\left(\bar{u}_{\text {dacn }} \cos n \phi\right. \\
& \left.\left.+\bar{u}_{\text {dasn }} \sin n \phi\right)\right] e^{i \frac{\omega}{\omega_{0}} \tau}
\end{aligned}
$$

where $N_{D}=(N-1) / 2$ for odd $N$ and $N_{0}=(N-2) / 2$ for even $N$, and $n$ is the nodal diameter index. Thus, the disk with the $\mathrm{N}$ attached blades can be characterized by $0,1,2, \ldots \mathrm{N}_{\mathrm{D}}$ and $\mathrm{N} / 2$ nodal diameters. The $\mathrm{N} / 2$ nodal diameter (second term in the square bracket of Eq. (2)) is present only for even N. Comparing Eqs. (1) and (2), it is clear that the traveling wave mode with the zero interblade phase angle, $\bar{u}_{\mathrm{da} 0}$, corresponds to the zero nodal diameter mode, $\bar{u}_{\text {daco } 0}$, that the traveling wave mode (with the interblade phase angle $=\pi$ ), $\vec{u}_{\text {daN/2 }}$, corresponds to the $\mathrm{N} / 2$ nodal diameter mode, and the traveling wave modes $\bar{u}_{d a r}$ and $\bar{u}_{d a(N-r)}$ combine to form two standing wave modes, $\bar{u}_{\text {dacr }}$ and $\bar{u}_{\text {dasr }}$. Thus, either a traveling or a standing wave mode approach can be used to describe the motion of a disk and the same is true of the blade or of the bladed-disk system (see also the discussion in Refs. 14, 16, 22 and 28). The above relationship will be used in deriving the blade aerodynamic loads due to the disk motion.

\section{Coordinate Systems}

Coordinate systems employed in deriving the equations of motion of the sth blade are shown in Figs. 1 and 2. The axis system $X_{\Omega}, Y_{\Omega}, Z_{\Omega}$ rotates with a constant angular velocity $\Omega$ about the $X_{\Omega}$-axis. The $Y_{\Omega}$-axis coincides with the undeformed elastic axis of the blade. The principal axis, $x$, of the blade cross section at any point on the elastic axis is inclined to the $X_{\Omega}$-axis by an angle $\xi$ as shown in Fig. 2 . The disk elastic deformation, $u_{d}$, and the blade deformations, $u, v, w$, and $\alpha$, translate and rotate the $x y z$ system to the $x_{3} y_{3} z 3$ system.

\section{Motion of a Tuned Bladed-Disk System}

Following common practice in the literature, the disk motion is expressed in standing wave modes and the blade motion is expressed, as in Ref. 22, in traveling wave modes. For the disk, using a single quadratic deflection shape along the radius for all the possible nodal diameter modes, the expression for the motion is given by Eq. (2) with

$$
R\left(\bar{n}_{d}\right)=\bar{n}_{d}^{2}
$$

For the blades, assume the number of modes retained in the plane of rotation, in the plane perpendicular to the plane of rotation, and in torsion are $M_{h}, M_{g}$, and $M_{\alpha}$, respectively. Then, the motion of the sth blade can be represented as

$$
\begin{aligned}
\bar{w} & =w / b_{R}=\sum_{j=1}^{M_{h}} w_{j}(\bar{n}) \bar{h}_{s j} e^{\left(i \frac{\omega}{\omega_{0}} \tau\right)} \\
& =\sum_{j=1}^{M_{h}} w_{j}(\bar{n}) \bar{h}_{a r j} e^{i\left(\beta_{r} s+\frac{\omega}{\omega_{0}} \tau\right)} \\
\bar{u} & =u / b_{R}=\sum_{j=1}^{M_{g}} w_{j}(\bar{n}) \bar{g}_{s j} e^{\left(i \frac{\omega}{\omega_{0}} \tau\right)}
\end{aligned}
$$

$$
+f_{c}(\vec{n}) \vec{u}_{d} \mid \begin{aligned}
& \bar{n}_{d}=1 \\
& \psi=\psi_{s}
\end{aligned}
$$

$$
=\sum_{j=1}^{M_{g}} w_{j}(\bar{n}) \bar{g}_{a r j} e^{i\left(\beta_{r} s+\frac{\omega}{\omega_{0}} \tau\right)}+\left.f_{c}(\bar{\eta}) \cdot \bar{u}_{d}\right|_{\begin{array}{l}
\bar{n}_{d}=1 \\
\psi=\psi_{s}
\end{array}} .
$$

$$
\alpha=\sum_{j=1}^{M_{\alpha}} A_{j}(\bar{\eta}) \alpha_{s j} e^{i \frac{\omega}{\omega_{0}} \tau}-\left.\frac{\partial \bar{u}_{d}}{\partial \phi}\right|_{\begin{array}{l}
\bar{n}_{d}=1 \\
\psi=\psi_{s}
\end{array}}
$$

$$
=\sum_{j=1}^{M_{\alpha}} A_{j}(\bar{n}) \alpha_{a r j} e^{i\left(\beta_{r} s+\frac{\omega}{\omega_{o}} \tau\right)}-\left.\frac{\partial \bar{u}_{d}}{\partial \phi}\right|_{\substack{\bar{n}_{d}=1 \\ \psi=\psi_{s}}}
$$


In Eqs. (4) to (6), the complex constants $\bar{h}_{s j}, \bar{g}_{s j}$ and a which are commonly used in fixed wing and rotary ${ }^{\text {fing }}$ aeroelasticity are expressed in terms of the new constants, $\bar{h}_{a r j}, \bar{g}_{a r j}$; and $\alpha_{a r j}$ and interblade phase angle since the aerodynamic loads are expressed in terms of the new constants. The total number of degrees of freedom for the bladed disk system is $N$ times $\left(1+M_{h}+M_{g}+M_{\alpha}\right)$. The

function $f(\bar{n})$ in Eq. (5) is determined by enforcing the continuity of displacement and slope at the blade-disk junction point

$$
\begin{aligned}
& \left.\bar{u}\right|_{\bar{n}=0}=\left.\frac{R_{H}}{b_{R}} \bar{u}_{d}\right|_{\bar{n}_{d}=1} \cdot \\
& \left.\frac{\partial \bar{u}}{\partial \bar{n}}\right|_{\bar{n}=0}=\left.\frac{R_{H}}{b_{R}} \frac{\partial \bar{u}_{d}}{\partial \bar{n}_{d}}\right|_{\overline{n_{d}}=1}
\end{aligned}
$$

These two conditions are satisfied if

$$
f_{C}(\bar{n})=\frac{R_{H}}{b_{R}}\left(1+2 \bar{n} \frac{L}{R_{H}}\right)
$$

In Eqs. (4) and (5), the standard nonrotating orthogonal normal modes in bending for a beam with fixed-free boundary conditions are given by

$$
\begin{aligned}
& w_{j}(\bar{n})=\cosh \left(p_{j} \bar{n}\right)-\cos \left(p_{j} \bar{n}\right) \\
& -\frac{\left(\cos p_{j}+\cosh p_{j}\right)}{\left(\sin p_{j}+\sinh p_{j}\right)}\left[\sinh \left(p_{j} \bar{n}\right)-\sin \left(p_{j} \bar{n}\right)\right] \\
& \text { where } p_{j} \text { is calculated from } \\
& \cos p_{j} \cosh p_{j}+1=0 .
\end{aligned}
$$

In Eq. (6) the orthogonal nonrotating modes in torsion for a uniform twisted beam with fixed-free boundary conditions are obtained from Ref. 25 and are

$$
\begin{aligned}
& A_{j}(\bar{n})=\left[\left(\sinh k_{1} \bar{n}-\frac{k_{1}}{k_{2}} \sin k_{2} \bar{n}\right)\right. \\
& \left.-\frac{\left(k_{1}^{2} \sinh k_{1}+k_{1} k_{2} \sin k_{2}\right)}{\left(k_{1}^{2} \cosh k_{1}+k_{2}^{2} \cos k_{2}\right)}\left(\cosh k_{1} \bar{n}-\cos k_{2} \bar{n}\right)\right]
\end{aligned}
$$

where

$$
k_{1}=\left[\left(\frac{F_{1}^{2}}{4}+F_{2}\right)^{1 / 2}+\frac{F_{1}}{2}\right]^{1 / 2}
$$

$$
\begin{aligned}
& k_{2}=\left[\left(\frac{F_{1}^{2}}{4}+F_{2}\right)^{1 / 2}-\frac{F_{1}}{2}\right]^{1 / 2} \\
& F_{1}=\frac{24}{1+u_{m}}\left(\frac{L}{c}\right)^{2}+\frac{4}{5} \frac{c^{2}}{t_{m}^{2}} \xi^{12}, \\
& F_{2}=12 \frac{\rho_{m}}{E_{m}} \frac{L^{4}}{t_{m}^{2}} \omega_{N R}^{2}
\end{aligned}
$$

The nonrotating frequency, wNR for each mode is obtained from

$$
\begin{aligned}
& 2 F_{2}+\left(2 F_{2}+F_{1}^{2}\right) \cos k_{2} \cosh k_{1}: \cdots \\
& +F_{1} \sqrt{F_{2}} \sin k_{2} \operatorname{sinhk_{1}=0}
\end{aligned}
$$

In calculating the mode shapes and frequencies, average values of actual blade shape are used for $\mathrm{L} / \mathrm{C}, \mathrm{c} / \mathrm{t}_{\mathrm{m}}$ and $\xi^{\prime}$ in Eq. (13).

\section{Motion of Mistuned Bladed-Disk System}

In the present formulation we consider mis. tuning only due to nonidentical structural properties of the blades, since the disk thickness is assumed to be uniform. Because of the mistuning there will be coupling between various traveling wave modes or equivalently: between the various standing wave modes. However; the general motion of an arbitrarily mistuned system can be expressed as a linear combination of the motions in either traveling or standing wave modes. The disk motion in Eq. (2) is already expressed in terms of the possible nodal diameter modes. The motion of the sth blade is obtained by simply superposing the blade deflections (in Eqs. (4) to (6)) in the rth traveling mode from $r=0$ to $r=N-1$.

Aerodynamic Model

For a rigid disk, the motion dependent aerodynamic lift and moment per unit blade span are expressed in Ref. 22 in terms of four coefficients $\ell$ hhr, $\ell_{\text {har, }} \ell_{\alpha h r}$, and $\ell_{a \alpha r}$ and blade motions which are written in a traveling form. These coefficients were calculated by using Smith's theory in subsonic flow and Adamczyk and Goldstein's theory in supersonic flow with a subsonic leading edge. At any radial station, the relative Mach number is a function of in-flow conditions and the rotor speed. Most current fan designs have supersonic flow at the blade tip and subsonic flow at the blade root. Consequently, some region of the blade encounters transonic flow. Since the above theories are not valid in the transonic region, the subsonic theory with Meff $=0.9$ for stations in the range $0.9<M$ <f $<\mathrm{I}$ and the supersonic theory with $M_{\text {eff }}=1.1$ for stations in the range $1.0 \leq M_{\text {eff }} \leq 1.1$ were used.

For a flexible disk, the lift and moment expressions can be divided conveniently into two parts: one due to blade motion and the other due to disk motion. The first part directly flows 
from Ref. 22. The second part can be obtained in two steps. First, express the lift and moment due to disk motion in the traveling wave form represented by Eq. (1) in terms of the four coefficients, mentioned earlier. Second, by taking the real part of the resulting expressions, by rearranging terms, and by assuming simple harmonic motion, derive the expressions for lift and moment per unit span due to disk motion represented by Eqs. (2) and (3) (see also refs. 14 and 28). Combining these two parts, the lift and moment expressions per unit span of the sth blade can be derived and are

$$
\begin{aligned}
& L_{a}=-\pi a_{a} b_{R^{\omega}}^{3} \sum_{r=0}^{N-1}\left[e _ { h h r } ( \frac { b } { b _ { R } } ) ^ { 2 } \left[\cos \xi \sum_{j=1}^{M_{h}} w_{j}(\bar{n}) h_{a r j}\right.\right. \\
& \left.+\sin \xi \sum_{j=1}^{M_{g}} w_{j}(\bar{n}) \bar{g}_{a r j}\right] \\
& \left.+\ell_{h a r}\left(\frac{b}{b_{R}}\right)^{3} \sum_{j=1}^{M_{a}} A_{j}(\bar{n}) a_{a r j}\right] e^{i\left(\frac{\omega}{\omega_{o}} \tau+B_{r} s\right)} \\
& +f_{c}(\bar{n}) \sin \xi\left(\frac{b}{b_{R}}\right)^{2}\left[e_{h h 0} \bar{u}_{d a c 0}\right. \\
& +\ell_{h h} \frac{N}{2} \cos \frac{N}{2} \Phi_{s} \bar{u}_{d a c} \frac{N}{2} \\
& +\sum_{n=1}^{N_{D}}\left[\left(\ell_{h h 1 n} \bar{u}_{d a c n}+\ell_{h h 2 n} \bar{u}_{d a s n}\right) \cos \psi_{s}\right.
\end{aligned}
$$$$
\left.\left.+\left(-\ell_{h h 2 n} \bar{u}_{d a c n}+\ell_{h h 1 n} \bar{u}_{\text {dasn }}\right) \sin n_{\psi} s\right]\right] e^{i \frac{\omega}{\omega_{0}} \tau}
$$$$
+\left(\frac{b}{b_{R}}\right)^{3} \sum_{n=1}^{N_{D}} n\left[\left(\ell_{h \alpha 1 n} \bar{u}_{d a s n}-\ell_{h \alpha 2 n} \bar{u}_{d a c n}\right) \cos n_{s}\right.
$$$$
\left.\left.-\left(\ell_{h \alpha 2 n} \bar{u}_{d a s n}+\ell_{h a 1 n} \bar{u}_{d a c n}\right) \sin n_{\psi} s\right]\right) e^{i \frac{\omega}{\omega_{0}} \tau}
$$

$$
\begin{aligned}
& M_{a}=\pi \rho a_{b} b^{4}{ }^{2} \sum_{r=0}^{N-1}\left[e _ { \alpha h r } ( \frac { b } { b _ { R } } ) ^ { 3 } \left[\cos \xi \sum_{j=1}^{M_{h}} w_{j}(\bar{n}) h_{a r j}\right.\right. \\
& \left.+\sin 5 \sum_{j=1}^{M_{g}} w_{j}(\bar{n}) \bar{g}_{a r j}\right] \\
& \left.+e_{\alpha a r} \frac{b}{b_{R}} \sum_{j=1}^{4} A_{j}(\vec{n}) a_{a r j}\right] e^{i\left(\frac{\omega}{\omega_{0}} \tau+\beta_{r} s\right)} \\
& +f_{c}(\bar{n}) \sin \xi\left(\frac{b}{b_{R}}\right)^{3}\left[e_{a h 0} \bar{u}_{d a c 0}\right. \\
& +e_{\alpha h \frac{N}{2}} \cos \frac{N}{2} \phi_{s} \bar{u}_{\text {dac }} \frac{N}{2} \\
& +\sum_{n=1}^{N_{D}}\left[\left(\ell_{\alpha h 1 n} \bar{u}_{d a c n}+\varepsilon_{\alpha h 2 n} \bar{u}_{d a s n}\right) \cos n \phi_{s}\right. \\
& \begin{array}{l}
\left.+\left(-e_{\alpha h 2 n} \bar{u}_{d a c n}+e_{\alpha h 1 n} \cdot \bar{u}_{d a s n}\right) \sin n_{\psi} s\right] e^{i \frac{\omega}{\omega_{0}} t} \\
+\left(\frac{b}{b_{R}}\right)^{4} \sum_{n=1}^{N_{D}} n\left[\left(\ell_{\alpha \alpha 1 n} \bar{u}_{d a s n}-\ell_{\alpha \alpha 2 n} \bar{u}_{d a c n}\right) \cos n \psi_{s}\right.
\end{array} \\
& \left.-\left(\ell_{\alpha \alpha 2 n} \bar{u}_{d a s n}+\ell_{\alpha \alpha 1 n} \bar{u}_{d a c n}\right) \sin n_{\psi}\right] e^{i \frac{\omega}{\omega_{0}}}
\end{aligned}
$$

where

$$
\begin{aligned}
& \ell_{h h 1 n}=\frac{1}{2}\left[\ell_{h h n}+\ell_{h h(N-n)}\right] \\
& \ell_{h h 2 n}=-\frac{j}{2}\left[\ell_{h h n}-\ell_{h h(N-n)}\right. \\
& \vdots \\
& \ell_{\alpha \alpha 2 n}=-\frac{i}{2}\left[\ell_{\alpha \alpha n}+\ell_{\alpha \alpha(N-n)}\right]
\end{aligned}
$$

The coefficients $\ell_{h h r}, \ell_{h a r}, \ell_{g h r}$, and $\ell_{\alpha \alpha r}$ are calculated for each value of $r$ for specified values of Mach number, reduced frequency, $s / c, \xi$, and a. Then, the corresponding coefficients

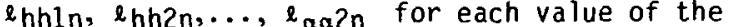
nodal diameter index $n$ are obtained from Eq. (17). Note that the subscripts $r$ and $n$ represent the interblade phase angle and nodal diameter number, respectively. 


\section{Structural Model}

The bladed-disk system is idealized as a uniform circular disk with constant thickness to which the blades are rigidly attached. Only the disk out of plane bending motion is considered. The blade model is the same as that used in Ref. 22 except for the fact that the warping of the blade cross sections is explicitly considered herein. Continuity and slope at the blade-disk junction is enforced as described in the previous section.

\section{Equations of Motion}

The strain energy expression for a circular isk 29

$$
\begin{aligned}
& U_{d}=\int_{0}^{2 \pi} \int_{0}^{R_{H}} \int_{D}\left[\left(\frac{\partial^{2} u_{d}}{\partial r^{2}}+\frac{1}{r} \frac{\partial u_{d}}{\partial r}+\frac{1}{r^{2}} \frac{\partial^{2} u_{d}}{\partial r^{2}}\right)^{2}\right. \\
& -2\left(1-v_{d}\right) \frac{a^{2} u_{d}}{\partial r^{2}}\left(\frac{1}{r} \frac{\partial u_{d}}{\partial r}+\frac{1}{r^{2}} \frac{\partial^{2} u_{d}}{\partial \phi^{2}}\right) \\
& +2\left(1-v_{d}\right)\left[\frac{\partial}{\partial r}\left(\frac{1}{r} \frac{\partial u_{d}}{\partial \phi}\right)\right]^{2} \\
& \left.+\frac{1}{2} t_{d}\left[\sigma_{r}\left(\frac{\partial u_{d}}{\partial r}\right)^{2 *}+\frac{\sigma_{\psi}}{r^{2}}\left(\frac{\partial u_{d}}{\partial \phi}\right)^{2}\right]\right) r d r d \psi
\end{aligned}
$$

where

$$
D=E_{d} t_{d}^{3} / 12\left(1-v_{d}^{2}\right)
$$

The stress components $\sigma_{r}$ and $\sigma_{\psi}$ are obtained by solving the following equations

$$
\begin{aligned}
& \frac{\partial \sigma_{r}}{\partial r}+\frac{\sigma_{r}-\sigma_{\psi}}{r}+\rho_{d} \Omega^{2} r=0 \\
& \ddot{\sigma}_{r}=\frac{E_{d}}{1-v_{d}^{2}}\left(E_{r}+\nu_{d} \varepsilon_{\psi}\right) \\
& \sigma_{\psi}=\frac{E_{d}}{1-v_{d}^{2}}\left(\varepsilon_{\psi}+v_{d} \varepsilon_{r}\right) \\
& \varepsilon_{r}=\frac{\partial u_{d}}{\partial r} \\
& \epsilon_{\psi}=\frac{u_{d}}{r} \\
& \left.u_{d}\right|_{r=0}=0 \\
& \sigma_{r} \sum_{r=R_{H}}=\frac{N}{2 \pi R_{H} t_{d}} \int_{R_{H}}^{R_{T} \cdot \vdots} m_{b} \Omega^{2} r \cdot d r
\end{aligned}
$$

and are

$$
\begin{aligned}
\sigma_{r} & =\frac{N}{2 \pi R_{H} t_{d}} \int_{R_{H}}^{\therefore} m_{b} \Omega^{2} r d r+\frac{3+v_{d}}{8} \Omega^{2} \rho_{d}\left(R_{H}^{2}-r^{2}\right) \\
\sigma_{\phi} & =\frac{N}{2 \pi R_{H} t_{d}} \int_{R_{H}}^{R_{T}} m_{b} \Omega^{2} r d r+\frac{3+v_{d}}{8} \Omega^{2} \rho_{d} R_{H}^{2} \\
& -\frac{1+3 v_{d}}{8} \Omega^{2} \rho_{d} r^{2}
\end{aligned}
$$

The strain energy of the $s^{\text {th }}$ blade follows from Ref. 22 and is

$$
\begin{aligned}
U & =\frac{1}{2} \cdot \int_{R_{H}}^{R}\left[E _ { m } \left[c_{1}{ }^{\prime \prime}+I_{x x}\left(u^{\prime \prime 2} \sin ^{2} \xi+w^{\prime \prime 2} \cos ^{2} \xi\right.\right.\right. \\
& \left.+2 u^{\prime \prime} w^{\prime \prime} \sin \xi \cos \xi\right)+I_{z z}\left(u^{\prime \prime 2} \cos ^{2} \xi+w^{\prime \prime} \sin ^{2} \xi\right. \\
& \left.-2 u^{\prime \prime} w^{\prime \prime} \sin \xi \cos \xi\right)+B_{1} \alpha^{2},^{2} \\
& -2 c_{2}{ }^{\prime \prime}\left(u^{\prime \prime} \sin \xi+w^{\prime \prime} \cos \xi\right) \\
& \left.\left.+2 B_{2}\left(u^{\prime \prime} \cos u-w^{\prime \prime} \sin \xi\right) \cdot \alpha^{\prime} \cdot\right]+G j \alpha\right] d r
\end{aligned}
$$

The explicit consideration of warping introduces two additional coefficients $C_{1}$ and $C_{2}$ and modifies the expression for $\mathrm{J}$

$$
\begin{aligned}
& c_{1}=\iint \lambda^{2} d x d z \\
& \therefore \quad C_{2}=\iint \lambda z d \dot{x} d z \\
& J=\iint\left[\left(z-\frac{\partial \lambda}{\partial x}\right)^{2}+\left(x+\frac{\partial \lambda}{\partial z}\right)^{2}\right] d x d z
\end{aligned}
$$

The other coefficients in Eq. (22) are defined in Ref. 22.

The kinetic energy of the disk is

$T_{d}=\frac{1}{2} \int_{0}^{2 \pi} \int_{0}^{R_{H}} \rho_{d} t_{d} \dot{u}_{d}^{2} r d r d \psi$

The kinetic energy of the $s^{\text {th }}$. blade also. follows from Ref. 22 and is 


$$
\begin{aligned}
T & =\frac{1}{2} \int_{R_{H}}^{R_{T}} m\left(\left(u^{2}+\dot{w}^{2}+k_{m^{\alpha}}^{2}{ }^{2}-2 e u \dot{a} \sin \xi\right.\right. \\
& +2 e \dot{w} \dot{a} \cos \xi) \\
& +\Omega^{2}\left[r^{2}-2 r U_{F}-2 r e\left(u^{\prime} \cos \xi-w^{\prime} \sin \xi\right)\right. \\
& -2 \text { rea }\left(u^{\prime} \sin \xi+w^{\prime} \cos \xi\right) \\
& +w^{2}+\left(k_{m_{2}}^{2}-k_{m_{1}}^{2}\right) \alpha^{2} \cos 2 \xi \\
& -\left(k_{m_{2}}^{2}-k_{m_{1}}^{2}\right) \alpha \sin 2 \xi \\
& +2 \text { eaw } \cos \xi-2 e w \sin \xi]\} d r
\end{aligned}
$$

The Coriolis and the inertial warping terms are considered to be small and are neglected.

The total strain and kinetic energies of the system are

$$
\left.\begin{array}{l}
U_{t}=U_{d}+\sum_{s=0}^{N-1} u \\
T_{t}=T_{d}+\sum_{s=0}^{N-1} T
\end{array}\right\}
$$

The generalized aerodynamic forces are calculated from the following virtual work expression

$$
\delta W=\sum_{s=0}^{N-1} \int_{R_{H}}^{R}\left(-L_{a} \sin \xi \delta u-L_{a} \cos \xi \delta w+M_{a} \delta \alpha\right) d r
$$

By substituting Eqs. (26) and (27) into Lagrange's equation, the equations of motion of the system are obtained. The final equations are nondimensionalized and cast in a standard eigenvalue form as

$$
[P]\{X\}=\gamma[Q]\{X\}
$$

where

$$
\begin{aligned}
& r=\left(\frac{\omega}{\omega_{0}}\right)^{2} \\
& {[Q]=[M]+[E D][A][E D]^{-1}} \\
& \{X\}=[E D]\{Y\} \\
& \qquad\left(\begin{array}{l}
\left\{\vec{u}_{d}\right\} \\
\left\{X_{0}\right\} \\
\left\{X_{1}\right\} \\
\vdots \\
\left\{X_{N-1}\right\}
\end{array}\right\} ;(Y\}=\left\{\begin{array}{l}
\left\{\bar{u}_{d}\right\} \\
\left\{Y_{0}\right\} \\
\left\{Y_{1}\right\} \\
\vdots \\
\left\{Y_{(N-1)}\right\}
\end{array}\right\} \\
& {[E O]=\left[\begin{array}{cc}
{[1]} \\
{[0]}
\end{array}\right]} \\
& {[E]}
\end{aligned}
$$

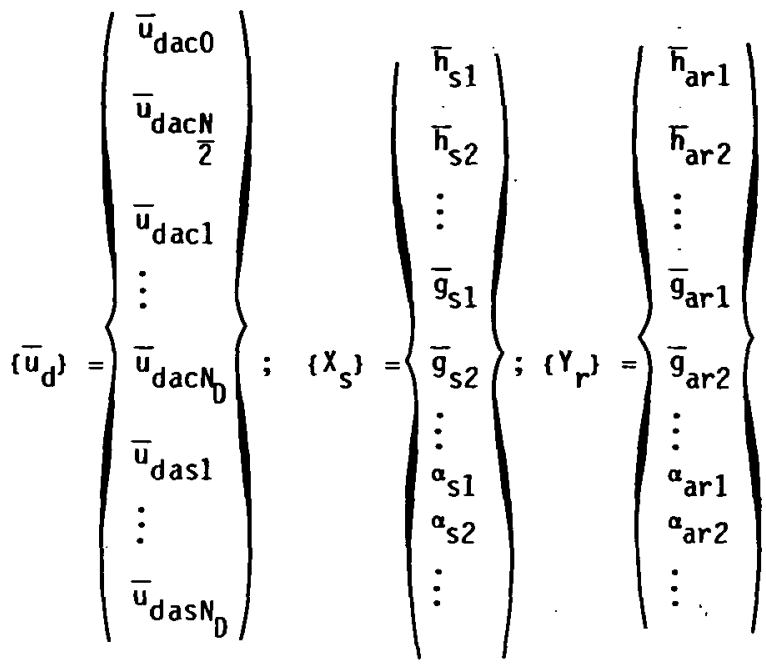

The matrices $[P],[M]$ and $[A]$ are the stiffness, mass and aerodynamic matrices of the system. The matrix [E] is defined in Ref. 22.

\section{Results and Discussion}

\section{Solution}

The flutter boundaries are obtained by solving the standard complex eigenvalue problem, Eq. (28). The relation between the frequency $\omega$ and $\gamma$ is

$$
i \frac{\omega}{\omega_{0}}=i \sqrt{r}=\bar{\mu} \pm i \bar{v}
$$

Flutter occurs when $\bar{\mu}>0$. Vibration characteristics are obtained by setting the aerodynamic matrix [A] to zero.

A computer program was written to assemble and solve the generalized eigenvalue problem. As mentioned earlier, the formulation and the program have very wide scope for investigating the effects of disk flexibility and blade frequency mistuning on both vibration and flutter of bladed disk assemblies. Because of space limitations, only selected results are presented.

\section{Vibration}

An advanced unshrouded fan stage (aspect ratio $=3.3$ ) representative of a next-generation $f$ an was chosen for analysis. This fan was analyzed in Ref. 22 by treating the disk as rigid. The disk of this fan consists of a thick hub, a thinner web and a short thick rim to house the blades. Accordingly, a disk model which consists of three concentric connected nonuniform annuli would be required for accurate modeling. However, to reduce the complexity of the model, the disk of the fan is idealized as a circular disk with uniform thickness. To investigate the effects of disk flexibility on both vibration and flutter, four values for disk thickness are considered. For descriptive purposes, the disk with thicknesses $1.891,0.1891$, 0.0946 and $0.0473 \mathrm{~m}$ are designated as very rigid, rigid, flexible, and very flexible disks, respectively. The equivalent thickness for a real practical fan may lie between the values for the rigid and flexible disk. The other properties of the 
disk are listed in Table 1. The blade properties are the same as those listed in Ref. 22 and hence they are not repeated here. The design speed of this 28-bladed fan stage is $4267-\mathrm{rev} / \mathrm{min}$.

The vibration analyses are performed by using six modes for each of the. 28 blades and all.pos-. sible nodal diameter modes for the disk. . The blade modes consist of two modes each in the plane of rotation, in the plane perpendicular, to the plane of rotation, and in torsion. The total number of degrees of freedom is $28 \times 6+28=196$ :

The blade frequencies with disk thickness $t_{d}=1.891 \mathrm{~m}$ are virtually the same as the blade cantilever frequencies. Thus, the disk with $t_{d}=1.891 \mathrm{~m}$ behaves :virtually like an infinitely rigid disk. Since the very rigid disk. case was analyzed in Ref. 22 for vibration and flutter, the results are not repeated herein. The variation of the frequency ratio with nodal diameter index is illustrated. in Figs: 3 .to 5 for the other three values of the disk thickness. . The frequency ratio is obtained by dividing the frequency by an arbitrarily chosen reference value, $\omega_{0}=200 \mathrm{~Hz}$. In these figures the frequency ratios are designated as $F, C, T$, and $D$ indicating that the blade flatwise, chordwise, torsion and disk motions, respectively, are predominant in the eigenvectors. The additional numbers 1 and 2 in the designations denote first and second modes. The blade cantilever frequencies are shown by dark symbols. Based on these frequency ratios and the corresponding eigenvectors, which.are not. shown because of space limitations, several interesting observations are made. (1) For a 28-bladed system, the identifica$t$ ion of the predominant motion in the eigenvector was found to be a tedious, job. This problem was solved:by analyzing a system with $4,8,12$ and 16 blades since the identification of predominant motion is easier for fewer blades. (2) For each nodal diameter, except for zero and fourteen, éach eigenvalue represents a pair of modes and each mode in the pair is a linear combination of the $\sin \psi_{\mathrm{s}}$ and cos $\mathrm{n}_{\mathrm{s}}$. "Furthermore, the modes in each pair are orthogonal as expected for a tuned system. The eigenvalues for the zero and the 14 th nodal diameter represent only one mode. In the zeroth nodal diameter mode all the blades move in phase and in the 14 th nodal diameter mode the phase difference between one blade and its neighbor is $180^{\circ} \ldots$ (3) Because of the pretwist of the blade, there is a coupling between blade flatwise and chordwise motions and between blade chordwise and disk motions. For the disk with $t_{d}=0.1891 \mathrm{~m}$, it can be seen from $F i g .3$, that the coupling between blade chordwise and the disk motions is stronger than that between blade flatwise and disk motions'. This is because the disk frequency is in the vicinity of the blade second chordwise frequency. This is contrary to assumptions often made in the published literature deal-. ing with bladed-disk vibrations. (4) Comparing the results in Figs. 3 to 5 , the disk frequency decreases with a decrease in disk stiffness, as should be expected. Furthermore, the coupling between the disk and blade motions and blade to. blade motions increases as the disk becomes thinner: Alternatively, one could say that "more of the disk modes couple with the blades modes as the disk flexibility decreases. (5), For the disk with $t_{f}=0.1891 \mathrm{~m}$, it can be seen from $F i g$. ' 3 that the coupling between the disk and blades for nodal diameters higher than four is almost negligible.
The effect of alternate mistuning on vibration is investigated. The method used here to vary the frequency from blade-to-blade is: simply to vary either the blade torsional stiffness $G J$ or the bending stiffiness $E_{m} I_{x x}$. For alternate mistuning the torsional st iffness of the odd numbered blades is increased by 10 percent and that of the even numbered blades is decreased by 10 percent from that of a tuned blade system: The result is a frequency variation : of approximately 7 percent. When this mistuning is introduced, the T-1 and T-2 modes in Figs. 3 to 5 are affected. The frequency ratios for the T-1 mode alternates between 1.06 and 1.13 and that for the T-2 mode between. 2.75 and 2.93.. The effect of mistuning in the other frequencies is very small.

Flutter of a Tuned Bladed-Disk.

The advanced fan stage arialyzed for vibrations is again considered to investigate flutter behavior including disk flexibility. The solution technique used herein to calculate the flutter boundary is the same as that described in Ref. 22. The correctness of the program was checked by comparing the flutter speed for the case with $t_{d}=1.891 \mathrm{~m}$. with that for the rigid disk case in Ref. 22 in-: cluding all the warping effects. Next, the effect of disk flexibility on flutter speed is illustrated in Fig. 6. The $f$ an stage is stable if the operating point is to the left of the boundary. For a disk with $t_{d}=0.1891 \mathrm{~m}$ the flutter boundary coincides with that for the tuned rigid disk $\left(t_{d}=1.891 \mathrm{~m}\right)$, indicating that this.amount of disk flexibility does not have any influence on flutter. For a disk thickness $t_{d}=0.0946 \mathrm{~m}$, the flutter speed is very slightly reduced. This reduction in flutter speed is more significant. when the disk thickness is reduced to $0.0473 \mathrm{~m}$. This degradation in flutter speed is expected because the coupling between disk and blade becomes very strong and reduces blade frequencies '(see. fig. 5). Additionally, the flutter mode is changed. from a predominantly torsional mode for the rigid disk to a predominantly bending mode for a very flexible disk. It should be mentioned that there is no blade bending-torsion coupling inherent in the blades themselves because the center of gravity and elastic axi.s of any blade are coincident. However, coupling between bending and torsional motions does occur because of the disk flexibility.

Furthermore, there is aerodynamic coupling between bending and torsional motions. 'Thus, flutter.' involving a predominantly bending mode is not surprising (see also. Ref. 16).

\section{Flutter of a Mistuned Bladed-Disk}

Previous publications $(16,17,22)$ have shown that the torsional: frequency mistuning could have a significant stabilizing effect on flutter. The effect of mistuning is further'investigated herein with the flexible disk model and some selected results are presented in Fig. 6. Alternate blade torsional frequency mistuning of approximately 7 percent has significantly.increased the flutter speed for the cases with $t_{d}=0.1891$, and $0.0946 \mathrm{~m}$. This finding is in agreement with that in Ref. 22 . Again, this amounts to saying that the disk flexibility does not have much influence on the flutter speed even in the presence of mistuning. Contrary to these two cases, there is no appreciable im- 
provement on flutter speed due to torsional frequency mistuning for the very flexible disk. This might be expected because the predominant motion in the flutter mode is bending rather than torsion. Next it is natural to ask whether an alternate bending frequency mistuning has any influence on flutter speed. To investigate this, the bending st iffness $E I_{m}$ of the. odd numbered blades was increased by 10 percent and that of the even numbered blades was decreased by 10 percent from that of a tuned blade. The result is a frequency variation of approximately 2.6 percent. With this mistuning the flutter boundaries for the very flexible disk is shown again in Fig. 6. Evidently, there is a small increase in flutter speed. However, this increase in flutter speed is not as great as that due to alternate torsional mistuning for the rigid disk. Again, it is concluded that mistuning has potential to increase the flutter speed even in the presence of mechanical coupling between blades introduced by disk flexibility. Qualitatively, these findings are in agreement with the corresponding ones in Ref. 21 where a modified typical section model was used. However, it should be emphasized that the flexibility of the disks which are employed for the current generation of turbofans does not have a significant effect on flutter speed. Still, the flexibility effects should be included in calculating forced response which is sensitive to small changes in system frequencies.

\section{Concluding Remarks}

An analytical model and an associated computer program to investigate the effects of disk flexibility on vibration and flutter characteristics of both tuned and mistuned bladed-disk assemblies are developed. From the parametric studies the following conclusions are reached.

(1) The flexibility of the disks which are employed for the current generation turbofans does not have much influence on either tuned or mistuned bladed-disk system flutter characteristics. Still, its effect may be significant on forced response which is sensitive to small changes in system frequencies.

(2) The flexibility of a very thin disk reduces flutter speed and changes the predominant character of the flutter mode from torsion to bending.

(3) Because of the blade pretwist, chordwise motion of the blades is more strongly coupled to the disk motion than is the blade flatwise motion. This is contrary to assumptions often made in the published literature dealing with bladed-disk vibrations.

(4) A moderate amount of blade frequency mistuning has significant potential to increase the flutter speed, even in the presence of the mechanical coupling between blades that is introduced by disk flexibility.

\section{References}

1. Wagner, J. T., "Coupling of Turbomachine Blade Vibrations Through the Rotor", Journal of Engineering for Power, Vol. 89, Oct. 1967, pp. 502-512.
2. Dye, R. C. F., and Henry, T..A., "Virbration Amplitudes of Compressor Blades Resulting from Scatter in Blade Natural Frequencies," Journal of Enginering for Power, Vol. 91, JuTy 1969.

3. Ewins, D. J., "Bladed Disc Vibration - A Review of Techniques and Characteristics", Recent Advances in Structural Dynamics, Univ. of Southamption, 1980, pp. 187-210.

4. El-Bayoumy, L. E. and Srinivasan, A: V., "Influence of Mistuning on Rotor-Blade Vibrations", AIAA Journal, Vol. 13, Apr. 1975, pp. 460-464.

5. Whitehead, D. S., "Effect of Mistuning on the Vibration of Turbomachine Blades Induced by Wakes," Journal of Mechanical Engineering Science, Vol. 8, Mar. 1966, pp. 15-21.

6. Whitehead, D. S., "Torsional Flutter of Unstalled Cascade Blades at Zero Deflections," ARC, R M 3429, 1964.

7. Hanamura, Y., and Tanaka, H., "A Modification of Flutter Characteristics by Changing Elastic Nature of Neighboring B.lades in Cascades," Tokyo Joint Gas Turbine Congress, Tokyo, Japan, 1977, pp. 418-427.

8. Ford, R. A. J., and Foord, C A., "An Analysis of Aeroengine Fan Flutter Using Twin Orthogonal Vibration Modes," ASME Paper 79-GT-126, Mar. 1979.

9. Hanamura, Y., "Flutter of Cascading Blade Row", Institute of Space and Aeronautical Science, University of Tokyo, Vol. 36, Report No. 459, 1971, pp. 1-36.

10. Shiori, J., "Non-Stall Normal Mode Flutter in Annular Cascades, Part 11-Experimental Study", Transactions of Japan Society of Aeronautical Engineering, Vol. 1, No. 1, 1958, pp. 26-45.

11. Carta, F. O., "Coupled Bladed-Disc-Shroud Flutter Instabilities in Turbo-Jet Engine Rotors," Journal of Engineering for Power, Vol. 89, July 1967, pp. 419-426.

12. Rao, B. M., and Kronenburger, L., Jr., "Aeroelastic Characteristics of a Cascade of Blades," Texas A\&M Univ., AFOSR-78-1027-TR, Feb. 1978, AD-A055 619.

13. Bendiksen, 0., and Friedmann, P.; "Coupled Bending-Torsion Flutter in Cascades", AIAA Journal, Vol. 18, No. 2, Feb. 1980, pp. 195-201.

14. Dugundji, J., "Flutter Analys is of a Tuned Rotor with Rigid and Flexible Disks," Mass. Institute of Technology, GT PDL Report No. 146, July 1979 .

15. Smith, G. C. C., and Elchuri, V., "Aeroelastic and Dynamic Finite Element Analyses of Bladed Shrouded Disks," Textron Bell Aerospace Co., Buffalo, N. Y., D2536-941001, (NASA CR159728) Mar. 1980 . 
16. Kaza, K. R. V., and Kielb, R. E., "Flutter and Response of a Mistuned Cascade in Incompressible Flow", AIAA Paper No. 81-0602, AIAA Journa 7 , Vol. 20, No. 8, Aug. 1982, pp. 1120-1127.

17. Kielb, R. E., and Kaza, K. R. V., "Aeroelastic Characteristics of a Cascade of Mistuned Blades in Subsonic and Supersonic Flows", ASME Paper 81-DET-122, Sept, 1980.

18. Srinivasan, A. V., and Fabunmi, J. A., "Cascade Flutter Analys is of Cantilevered Blades," Journal of Engineering for Gas Turbines and Power, Vol. 106, No, 1, Jan. 1984, pp. 34-43.

19. Bendiksen, 0. 0., "Flutter of Mistuned Turbomachinery Rotors," Journal of Engineering for Gas Turbines and Power, Vol. 106, No. 1, Jan. 1984, pp. 25-33.

20. Srinivasan, A. V., "Influence of Mistuning on Blade Torsional Flutter," United Technologies Research Center, East Hartford, Conn. R80-914545-1b, Aug. 1980 (NASA CR-165131).

21. Kielb, R. E., and Kaza, K. R. V., "Effects of Structural Coupling on Mistuned Cascade Flutter and Response," ASME Paper 83-GT-117, Journal of Engineering for Gas Turbines and Power, Vo1. 106, No. 1, Jan:, 1984, pp 17-24.
22. Kaza, K. R. V., and Kielb, R. E., "Coupled Bending-Bending-Torsion Flutter of a Mistuned Cascade with Nonuniform Blades", AIAA Paper No. 82-0726, May 1982.

23. Mehmed, 0., Kaza, K. R. V., Lubomski, J. F., and Kielb, R. E., "Bending-Torsion Flutter of a Highly Swept Advanced Turboprop", 1982 Aerospace Congress and Exposition, Anaheim, California, October 25-28, 1982.

24. Houbolt, J. C., and Brooks, G. W., "Differential Equations of Motion for Combined Flapwise Bending, Chordwise Bending, and Torsion of Twisted Nonuniform Rotor Blades", NACA Tech. Report 1346, 1958.

25. Kaza, K. R. V., and Kielb, R. E., "Effects of Warping and Pretwist on Torsional Vibration of Rotating Beams", Accepted for publication in the ASME Journal of Applied Mechanics.

26. Smith, S. N., "Discrete Frequency Sound Generation in Axia1 Flow Turbomachines", ARC ReM $3709,1973$.

27. Adamczyk, J. J., and Goldstein, M. E., "Unsteady Flow in a Supersonic Cascade with Subsonic Leading-Edge Locus," AIAA Journal, Vol. 16, No 12, Dec. 1978, pp. 1248-1254.

28. Dugundji, J. and Bundas, D. J., "Flutter and Forced Response of Mistuned Rotors Using Standing Wave Analysis", AIAA Paper 83-0845, 1983.

29. Timoshenko, S. A., Theory of Plates and Shel1s, McGraw-Hi11, New York, 1940. 
TABLE I. - DISK PROPERTIES

Radius, $R_{H}, \mathrm{~m} . \ldots . . . . . .0 .3876$

Thickness, $t_{d} . . . . . . . .2$ varied

Young's Modulus, $\mathrm{E}_{\mathrm{d}}, \mathrm{N} / \mathrm{m}^{2}, \ldots . . .0 .1234$

Material density, $\rho_{\mathrm{d}}, \mathrm{Kg} / \mathrm{m}^{3} \ldots \ldots . . . .4373$

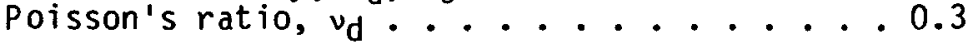




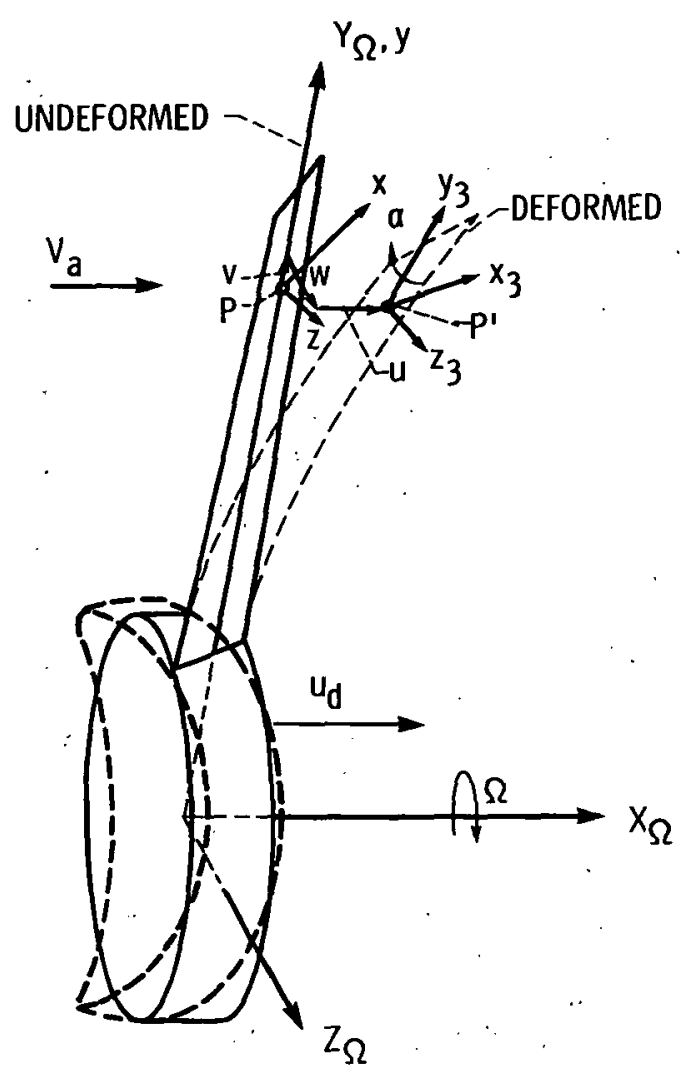

Figure 1. - Blade coordinate systems before and after deformation.

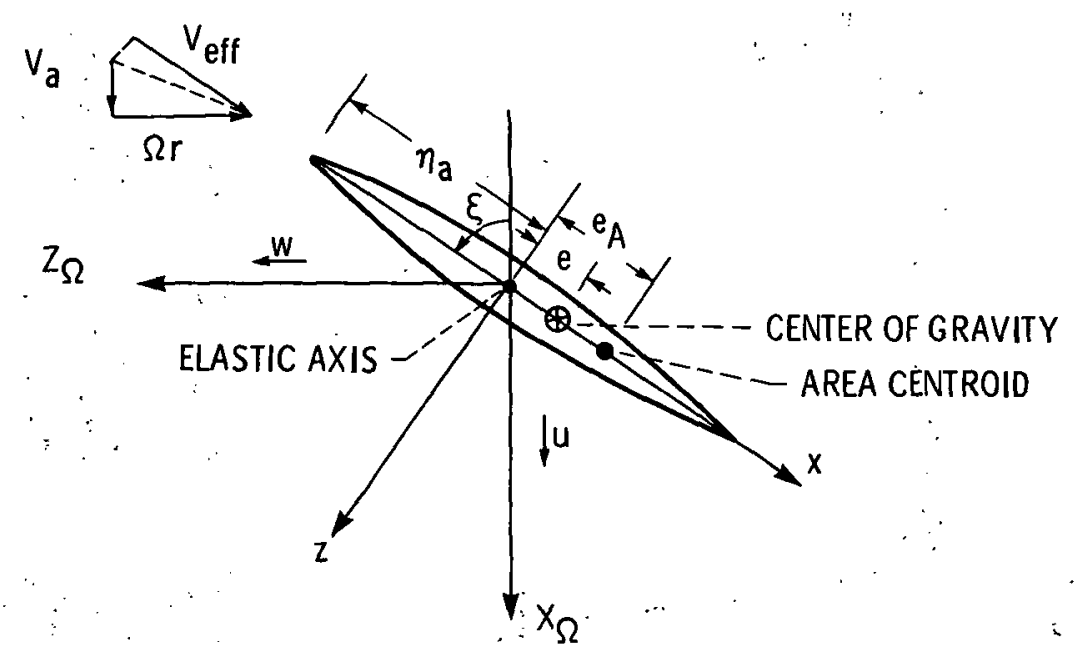

Figure 2. - Coordinate systems of blade cross section. 


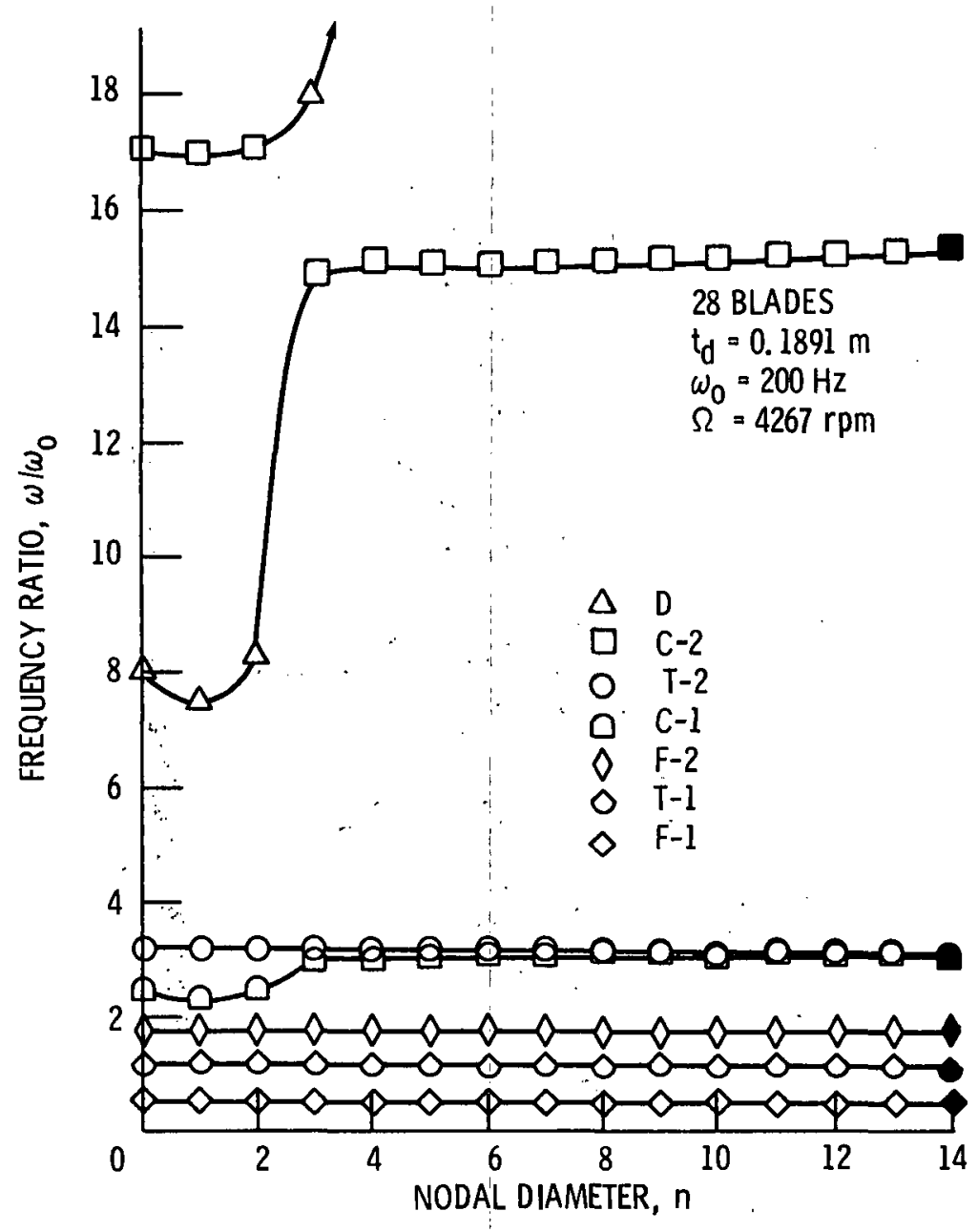

Figure 3. - Frequencies of bladed-disk system.

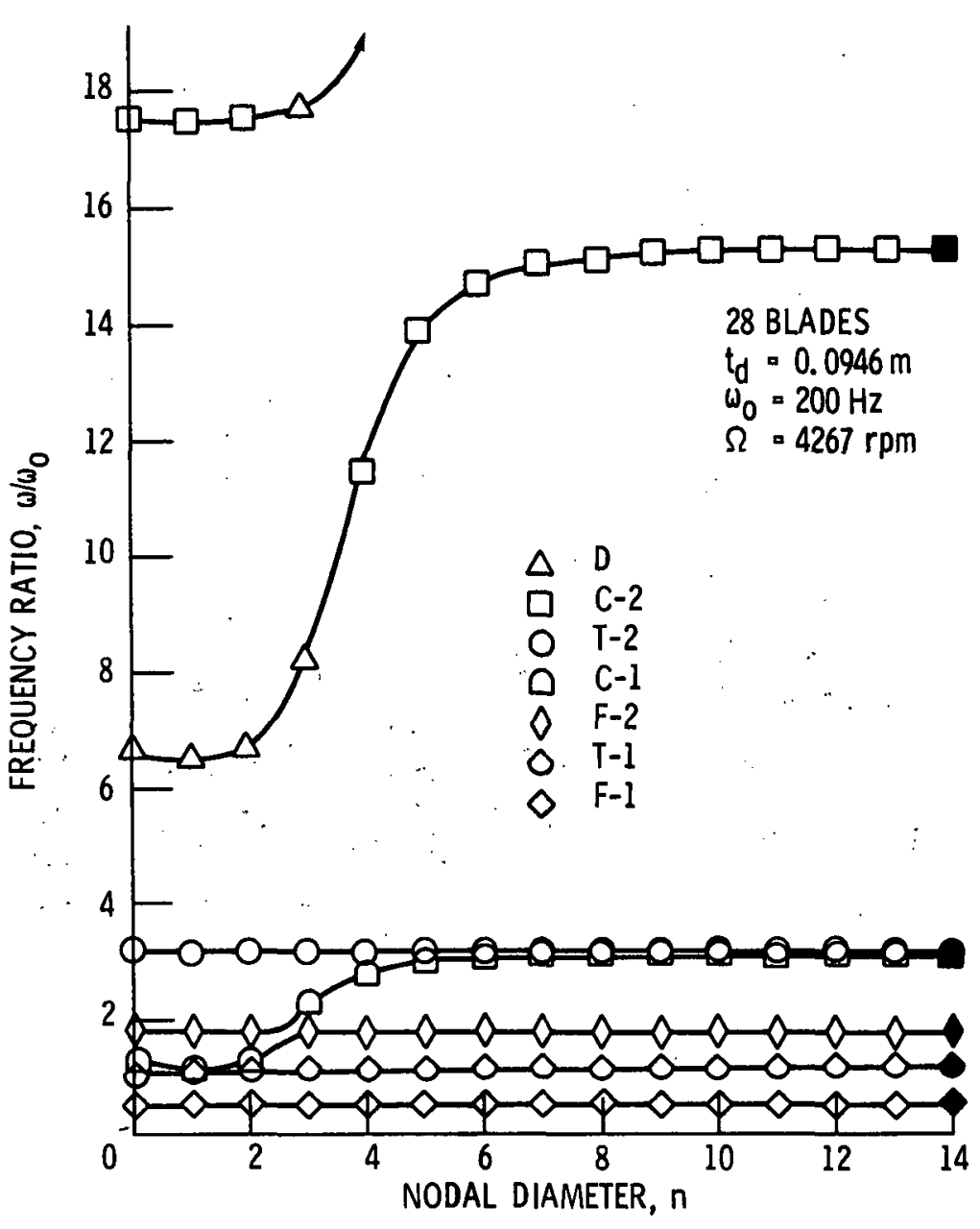

Figure 4. - Frequencies of bladed-disk system. 


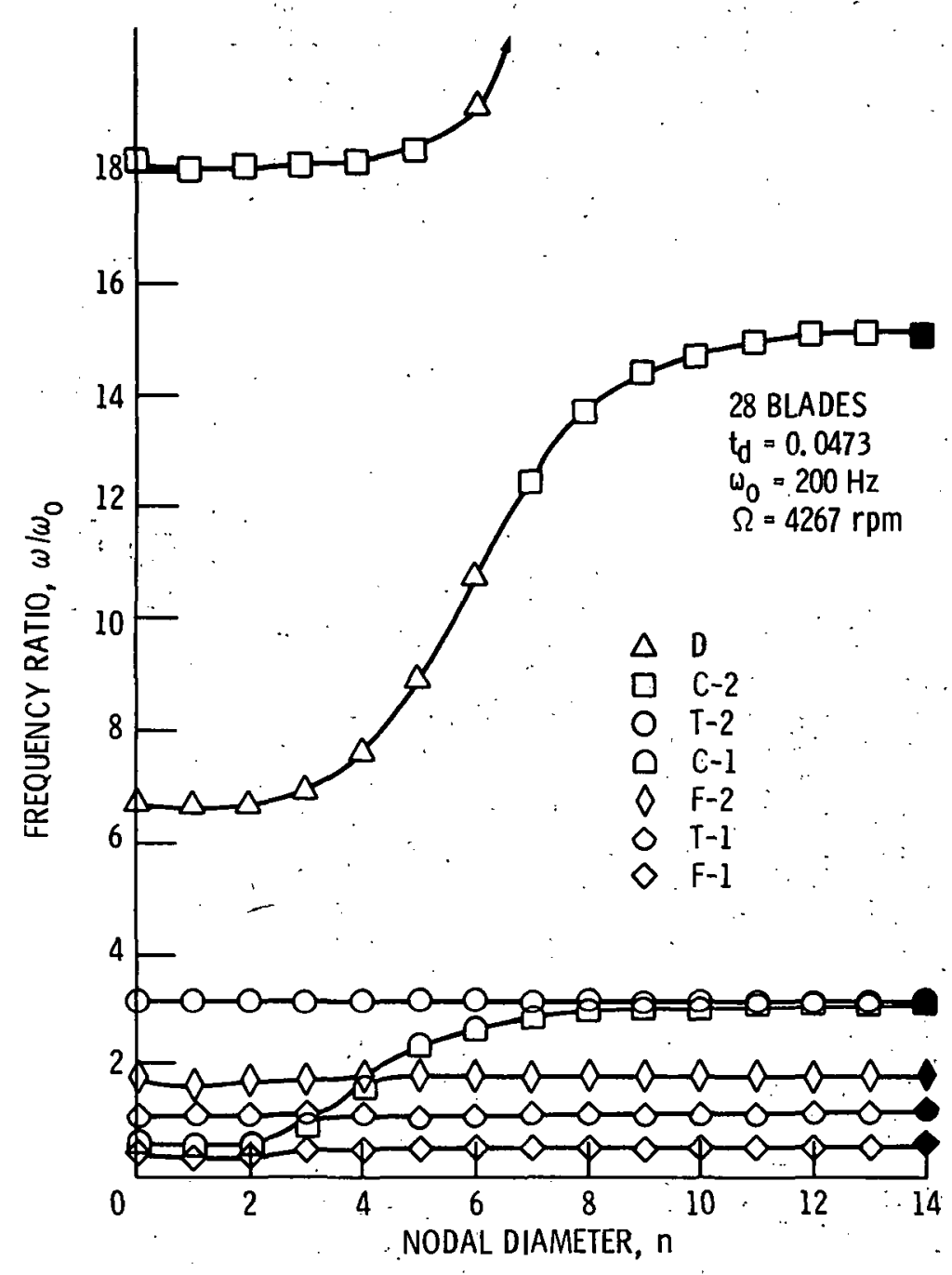

Figure 5. - Frequencies of bladed-disk system:

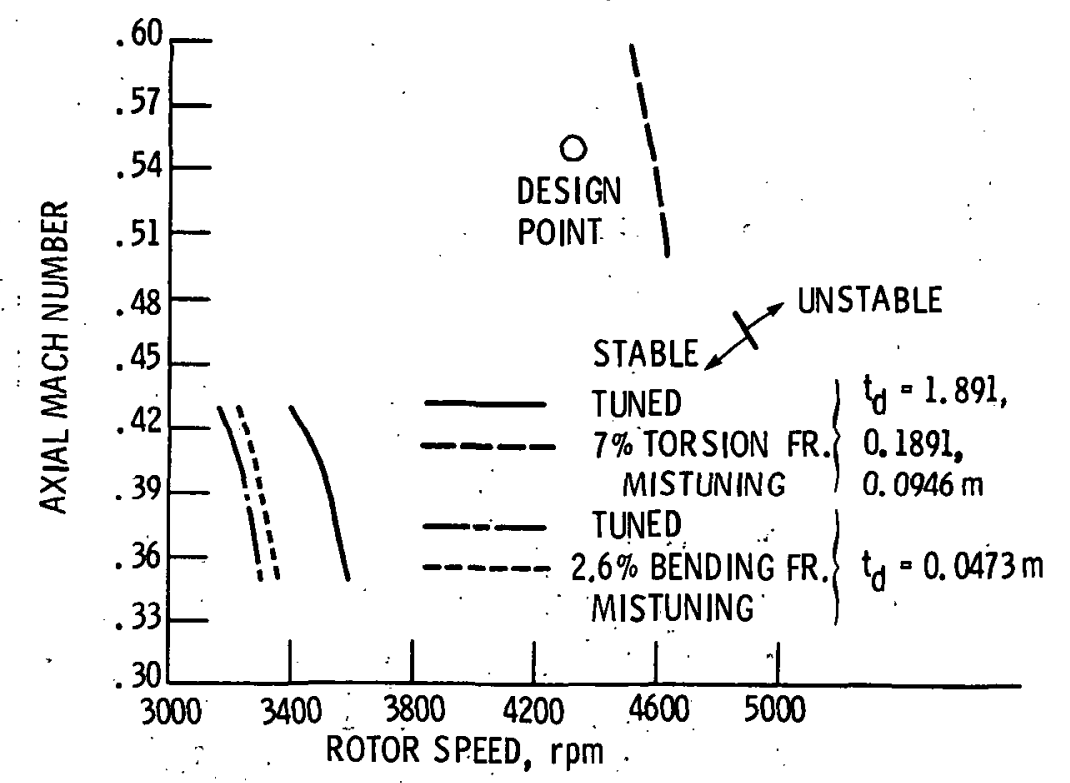

Figure 6. - Effect of disk flexipility on tuned and mistuned bladed-disk system. 


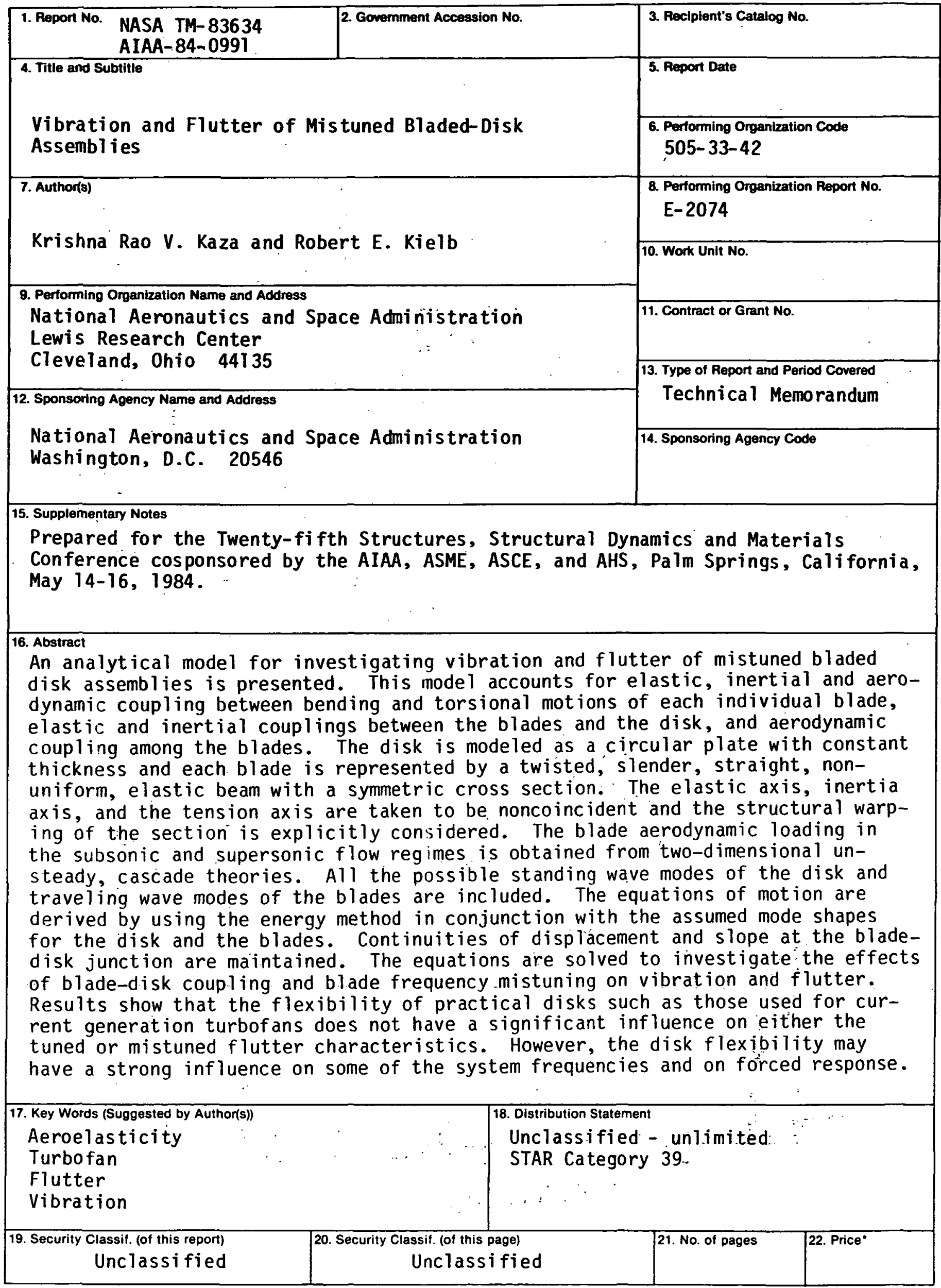

-For sale by the National Technical Information Service, Springfield, Virginia 22161 
National Aeronautics and Space Administration

Washington, D.C.

20546

Official Business

Penalty for Private Use, $\$ 300$
SPECIAL FOUATH CLASS MAIL BooK

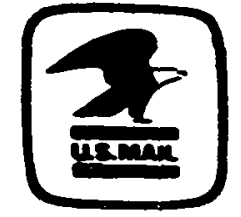

Portage and fees Paid Netional Aeronsutics and Spen Administrotion MASA-451 